\title{
Telomere length shortening is associated with treatment-free remission in chronic myeloid leukemia patients
}

\author{
Giovanni Caocci ${ }^{1 *}$ D, Marianna Greco ${ }^{1}$, Giuseppe Delogu ${ }^{2}$, Christian Secchi ${ }^{2,3}$, Bruno Martino ${ }^{4}$, Claudia Labate ${ }^{4}$, \\ Elisabetta Abruzzese ${ }^{5}$, Malgorzata Monika Trawinska ${ }^{5}$, Sara Galimberti ${ }^{6}$, Federica Orru' ${ }^{1}$ Claudio Fozza 7 , \\ Carlo Gambacorti Passerini ${ }^{8}$, Francesco Galimi ${ }^{2,3}$ and Giorgio La Nasa'
}

\begin{abstract}
We studied telomere length in $32 \mathrm{CML}$ patients who discontinued imatinib after achieving complete molecular remission and 32 age-sex-matched controls. The relative telomere length (RTL) was determined by q-PCR as the telomere to single copy gene (36B4) ratio normalized to a reference sample (K-562 DNA). Age-corrected RTL (acRTL) was also obtained. The 36-month probability of treatment-free remission (TFR) was $59.4 \%$. TFR patients showed shorter acRTL compared to relapsed (mean $\pm \mathrm{SD}=0.01 \pm 0.14$ vs $0.20 \pm 0.21 ; p=0.01$ ). TFR was significantly higher in CML patients with acRTL $\leq 0.09$ (78.9 vs $30.8 \%, p=0.002$ ). CML stem cells harboring longer telomeres possibly maintain a proliferative potential after treatment discontinuation.
\end{abstract}

Keywords: Chronic myeloid leukemia, Telomere, Treatment-free remission, Imatinib, Telomerase

Telomeres are specialized nucleoprotein structures composed of long arrays of TTAGGG repeats localized at the ends of human chromosomes able to maintain genome stability and integrity and to protect the cell from progressive DNA shortening during repeated division [1]. Telomere biology has been more extensively studied in chronic myeloid leukemia (CML) than in any other blood cancer. Shorter telomeres have been associated with CML, disease progression, poor prognosis, higher Hasford score, and acquisition of cytogenetic aberrations [2-4]. As yet, no studies have considered the possible association between telomere length and treatment-free remission (TFR) after discontinuation of therapy with tyrosine kinase inhibitors (TKIs).

Thirty-two chronic-phase CML patients discontinued TKI treatment after achieving complete molecular remission (CMR) for at least 18 months. All patients received imatinib therapy for more than 24 months. Two patients underwent second-line treatment with nilotinib

\footnotetext{
* Correspondence: giovanni.caocci@unica.it

${ }^{1}$ Hematology Unit, Department of Medical Sciences, Bone Marrow Transplant Center, R. Binaghi Hospital, University of Cagliari, Ospedale "R. Binaghi", Via Is Guadazzonis, 3, 09126 Cagliari, Italy

Full list of author information is available at the end of the article
}

because of molecular relapse. The median follow-up after discontinuation was 30 months (range 18-60). A complete molecular response was defined as undetectable breakpoint cluster region-Abelson (BCR/ABL1) by real-time quantitative polymerase chain reaction (qRTPCR) with a sensitivity of the assay corresponding to molecular response (MR)4 and MR4.5. Peripheral blood samples from 32 age- and sex-matched healthy individuals were used for control purposes. The relative telomere length (RTL) was determined by q-PCR according to the technique described by Cawthon in 2002 [5] (Additional file 1). Age-corrected RTL (acRTL) represented the difference in telomere length between patients and age- and sex-matched controls.

The characteristics of 32 chronic phase CML patients are shown in Table 1 . Thirteen patients (41\%) showed loss of CMR. All relapsed patients regained CMR after restarting treatment with TKIs. The 36-month cumulative probability of TFR was $59.4 \%$. RTL was assessed at a mean of 26 months from discontinuation (range 20-30). RTL was assessed at a mean of 26 months from discontinuation (range 18-30). The majority of relapses occurred within 9 months of therapy interruption (mean 8.7 months, range 2-20). In these 
Table 1 Characteristics of 32 CML patients according to treatment-free remission (TFR) or molecular relapse after imatinib discontinuation

\begin{tabular}{|c|c|c|c|c|c|}
\hline & Patients in TFR no. 19 (59 \%) & & Relapsed patients no. 13 (41\%) & & $p$ \\
\hline Age at diagnosis (mean, range) & $74(47-88)$ & & $56(37-77)$ & & 0.004 \\
\hline Leukocytes at diagnosis $\times 10^{3} / \mathrm{uL}$ (mean, range) & $50.47(8.15-221)$ & & $69(19.8-263)$ & & ns \\
\hline Platelets at diagnosis $\times 10^{3} / \mathrm{uL}$ (mean, range) & $472(178-918)$ & & $357(171-695)$ & & ns \\
\hline Months to CMR (median, range) & $28(3-88)$ & & $30(6-93)$ & & ns \\
\hline Months of TKls (median, range) & $86(24-127)$ & & $84(45-143)$ & & ns \\
\hline Months of TKls >60 (no., \%) & 13 & 68.4 & 12 & 92.3 & ns \\
\hline Male gender (no., \%) & 16 & 84.2 & 8 & 61.5 & ns \\
\hline \multicolumn{6}{|l|}{ Sokal risk (no., \%) } \\
\hline Low & 6 & 31.6 & 8 & 61.5 & ns \\
\hline Intermediate & 10 & 52.6 & 3 & 23.1 & ns \\
\hline High & 3 & 15.8 & 2 & 15.4 & ns \\
\hline First-line TKI treatment (no., \%) & 11 & 57.9 & 11 & 84.6 & ns \\
\hline Previous IFN treatment (no., \%) & 8 & 42.1 & 2 & 15.4 & ns \\
\hline Imatinib first-line TKI treatment (no., \%) & 19 & 100 & 13 & 100 & ns \\
\hline Nilotinib second-line TKI treatment (no., \%) & 1 & 5.3 & 1 & 7.7 & ns \\
\hline Age-corrected relative telomere length (mean \pm SD) & $0.01 \pm 0.14$ & & $0.20 \pm 0.21$ & & 0.01 \\
\hline
\end{tabular}

pres present, CMR complete molecular response, TKIs tyrosine kinase inhibitors, IFN interferon, ns not significant, SD standard deviation

patients, RTL was assessed after relapse. Overall, median RTL was slightly shorter in patients than in controls $(0.97$ vs 1.05$)$. The median value of acRTL in the CML cohort was 0.09 (range $-0.26,+0.86$ ). The Mann-Whitney $U$ test showed shorter acRTL in TFR patients compared to patients with molecular relapse (mean $\pm \mathrm{SD}=0.01 \pm 0.14$ vs $0.20 \pm 0.21 ; p=0.01$ ) (Additional file 2). Although the male gender was more frequent in TFR patients, we did not find any significant difference in telomere length between male and female. Patients were stratified according to the median value of acRTL $\leq 0.09$. TFR was significantly higher in CML patients with acRTL $\leq 0.09$ in comparison to those with longer telomeres (78.9 vs $30.8 \%, p=0.002$ ) (Fig. 1).

Previous studies suggest a dual-step model for telomere length changes in CML. In the earlier phases, increased turnover of Philadelphia positive $(\mathrm{Ph}+)$ progenitors would result in telomere shortening, leading to genetic instability. Later, CML cells would escape senescence and apoptosis through upregulation of telomerase and restored telomere length. This would promote the occurrence of genetically unstable CML subclones with a selective growth advantage [6]. Discontinuation of TKIs is the next hurdle to be overcome in the management of CML patients. Several factors have been identified as potentially capable of predicting durable TFR and hopefully definitive recovery $[7,8]$. A significant correlation between younger age and molecular relapse was reported [9]. In our study, CML patients had a slightly shorter telomere length than healthy controls and we found a statistically significant correlation between aging and telomere shortening. However, the most interesting finding was that TFR patients showed significantly shorter acRTL compared to molecular relapses. A possible explanation is that quiescent CML stem cells harboring longer telomeres somehow manage to escape senescence mechanisms and maintain a proliferative

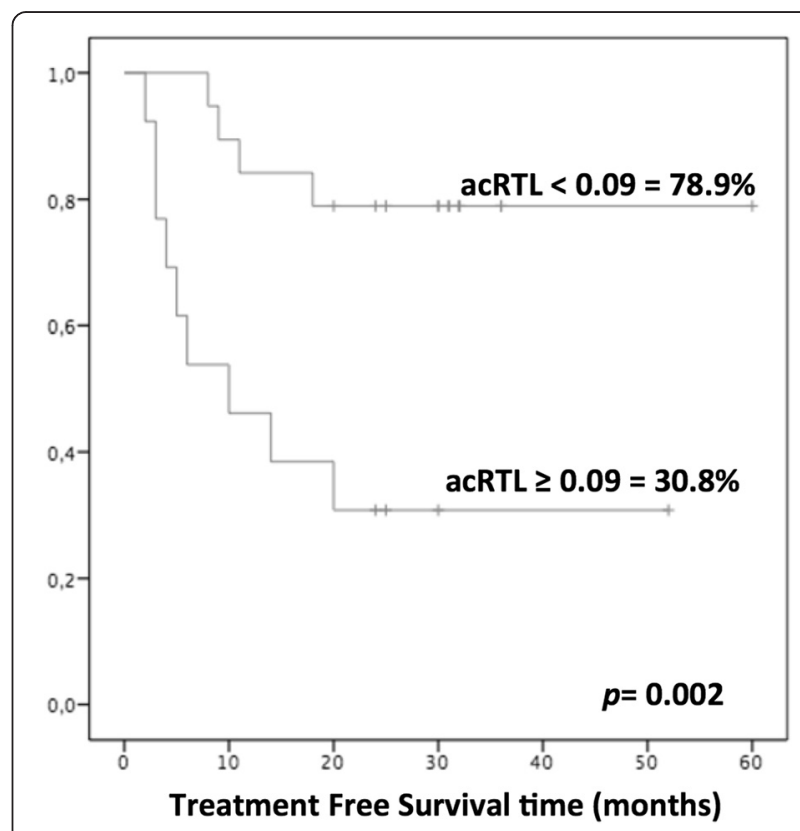

Fig. 1 Treatment-free remission (TFR) according to age-corrected relative telomere length (acRTL) $\geq 0.09$ in $32 \mathrm{CML}$ patients 
potential even after discontinuation of imatinib treatment, but this hypothesis should be supported by CML stem cell telomere assessment in patients with molecular response. Some limitations need to be noted in our study. First, the cohort of patients was relatively small and a longitudinal telomere assessment from diagnosis is lacking. Furthermore, we did not determine sorted myeloid compartment telomere length, but previous reports showed that no significant differences in CML telomere lengths are observed when comparing peripheral mononuclear blood cells, fractionated peripheral neutrophils, and non-fractionated bone marrow mononuclear cells [10]. The present study is the first to suggest that patients with longer telomeres would seem to be more susceptible to relapse after TKI treatment.

\section{Additional files}

Additional file 1: Additional methods. (DOCX $38 \mathrm{~kb}$ )

Additional file 2: Boxplot of age-corrected relative telomere length (acRTL) in the group of 19 treatment-free remission (TFR) and 13 molecular-relapsed CML patients. Mean \pm SD $=0.01 \pm 0.14$ vs $0.20 \pm 0.21$; $p=0.01$. (TIFF $148 \mathrm{~kb}$ )

\section{Abbreviations}

$\mathrm{CML}$, chronic myeloid leukemia; TFR, treatment-free remission; CMR, complete molecular remission; MR, molecular response; qRT-PCR, real-time quantitative polymerase chain reaction; $B C R / A B L 1$, breakpoint cluster regionAbelson; TKls, tyrosine kinase inhibitors; RTL, relative telomere length; $\mathrm{aCRTL}$, age-corrected relative telomere length; WBC, white blood cell; PLT, platelets

\section{Acknowledgements}

We are deeply grateful to the patients who participated in this study. We also wish to thank Anna Maria Koopmans for her professional writing assistance.

\section{Funding}

The authors did not receive any financial support.

\section{Availability of data and materials}

DNA samples are available at CTMO-Ospedale Binaghi, Università di Cagliari.

\section{Authors' contributions}

GC, MG, FO, and GLN participated in the conception and design of the study. GC, MG, GD, CS, BM, CL, EA, MMT, SG, FO, CF, CGP, FG, and GLN contributed to the collection and assembly of the data. GC, MG, and GD carried out the statistical analysis. GC, MG, FO, and GLN contributed to the manuscript writing. All authors approved the final manuscript.

\section{Competing interests}

The authors declare that they have no competing interests.

\section{Consent for publication}

Not applicable.

\section{Ethics approval and consent to participate}

Approval for the study was obtained from the competent Ethics Committee of Rome, Reggio Calabria and Cagliari; enrolled patients were informed of the potential risks and benefits of stopping TKI treatment.

\section{Author details}

'Hematology Unit, Department of Medical Sciences, Bone Marrow Transplant Center, R. Binaghi Hospital, University of Cagliari, Ospedale "R. Binaghi", Via Is Guadazzonis, 3, 09126 Cagliari, Italy. ${ }^{2}$ Department of Biomedical Sciences,
University of Sassari, Sassari, Italy. ${ }^{3}$ Istituto Nazionale Biostrutture e Biosistemi, University of Sassari, Sassari, Italy. ${ }^{4}$ Division of Hematology, Ospedali Riuniti, Reggio Calabria, Italy. ${ }^{5}$ Hematology, S. Eugenio Hospital, University of Tor Vergata, Rome, Italy. ${ }^{6}$ Department of Clinical and Experimental Medicine, Section of Hematology, University of Pisa, Pisa, Italy. ${ }^{7}$ Department of Clinical and Experimental Medicine, University of Sassari, Sassari, Italy. ${ }^{8}$ Department of Internal Medicine, University of Milano Bicocca, Monza, Italy.

Received: 8 June 2016 Accepted: 25 July 2016

Published online: 29 July 2016

\section{References}

1. Blackburn EH. Structure and function of telomeres. Nature. 1991;350:569-73.

2. Lobetti-Bodoni C, Ferrero D, Genuardi E, et al. Telomere loss in Philadelphianegative hematopoiesis after successful treatment of chronic myeloid leukemia: evidence for premature aging of the myeloid compartment. Mech Ageing Dev. 2012;133:479-88.

3. Boultwood J, Fidler C, Shepherd P, et al. Telomere length shortening is associated with disease evolution in chronic myelogenous leukemia. Am J Hematol. 1999:61:5-9.

4. Drummond M, Lennard A, Brummendorf T, Holyoake T. Telomere shortening correlates with prognostic score at diagnosis and proceeds rapidly during progression of chronic myeloid leukemia. Leuk Lymphoma. 2004:45:1775-81.

5. Cawthon RM. Telomere measurement by quantitative PCR. Nucleic Acids Res. 2002;30:e47.

6. Brummendorf $\mathrm{TH}$, Holyoake $\mathrm{TL}$, Rufer $\mathrm{N}$, et al. Prognostic implications of differences in telomere length between normal and malignant cells from patients with chronic myeloid leukemia measured by flow cytometry. Blood. 2000:95:1883-90.

7. Caocci G, Martino B, Greco M, et al. Killer immunoglobulin-like receptors can predict TKI treatment-free remission in chronic myeloid leukemia patients. Exp Hematol. 2015:43:1015-8.

8. Rousselot P, Charbonnier A, Cony-Makhoul P, et al. Loss of major molecular response as a trigger for restarting tyrosine kinase inhibitor therapy in patients with chronic-phase chronic myelogenous leukemia who have stopped imatinib after durable undetectable disease. J Clin Oncol. 2014; 32:424-30.

9. Mori S, Vagge E, le Coutre $P$, et al. Age and $\mathrm{dPCR}$ can predict relapse in CML patients who discontinued imatinib: the ISAV study. Am J Hematol. 2015;90:910-4

10. Iwama H, Ohyashiki K, Ohyashiki JH, et al. The relationship between telomere length and therapy-associated cytogenetic responses in patients with chronic myeloid leukemia. Cancer. 1997:79:1552-60.
Submit your next manuscript to BioMed Central and we will help you at every step:

- We accept pre-submission inquiries

- Our selector tool helps you to find the most relevant journal

- We provide round the clock customer support

- Convenient online submission

- Thorough peer review

- Inclusion in PubMed and all major indexing services

- Maximum visibility for your research

Submit your manuscript at www.biomedcentral.com/submit
C Biomed Central 